\title{
IF FIBER OPTICS AND DIGITAL CORRELATING SPEC- TROMETER ON THE JCMT-CSO INTERFEROMETER
}

\author{
O.P. LAY AND R.E. HILLS \\ Mullard Radio Astronomy Observatory, Cambridge, England
}

J. CARLSTROM

Astronomy Department, California Institute of Technology, Pasadena, California

\section{INTRODUCTION}

This paper describes the delay lines designed and built for the JCMT-CSC: submillimeter interferometer located on the summit of Mauna Kea, Hawaii and described in the paper by Carlstrom et al. in these proceedings.

One of the major features of the project is the use of fiber optics instead of coaxial cable to bring the IF signals $(1-2 \mathrm{GHz})$ from the receivers to the spectral line correlator via a set of fiber optic delay lines. Optical fiber now has many advantages over coax including very large bandwidth, low loss, economical cost and size and immunity to electrical interference.

Soares et al. (these proceedings) describe a delay line system that can be used with a broadband continuum correlator requiring very fine delay steps. The JCMT-CSO interferometer was intended to be used primarily with a digital correlating spectrometer that has a range of built-in delays, greatly easing the demand on delay line resolution.

\section{THE CORRELATOR}

The correlator used is a Digital Autocorrelation Spectrometer (DAS) built by Albert Bos at Dwingeloo, Holland. Already in use at JCMT as a single dish spectral line correlator, it is also capable of cross correlation. Its 2048 separate lags are configurable in up to eight independent sub-bands. The resulting 1024 complex frequency channels cover bandwidths ranging from $125 \mathrm{MHz}$ to $1 \mathrm{GHz}$.

\section{DESIGN AND OPERATION}

The purpose of the delay lines is to equalize the two path lengths from the correlator to the source. However the DAS forms cross correlations over a number of different relative delays and it is sufficient to ensure that the peak of the correlation function (the "white light fringe") is roughly centred in the range of delays available in the correlator (the "lag window"). Any offset manifests; itself as a phase gradient in the frequency spectrum and can be removed by software post-processing at the expense of a small loss in amplitude. Calculations show that this amplitude loss is a function of spectral linewidth and the loss is 
a)

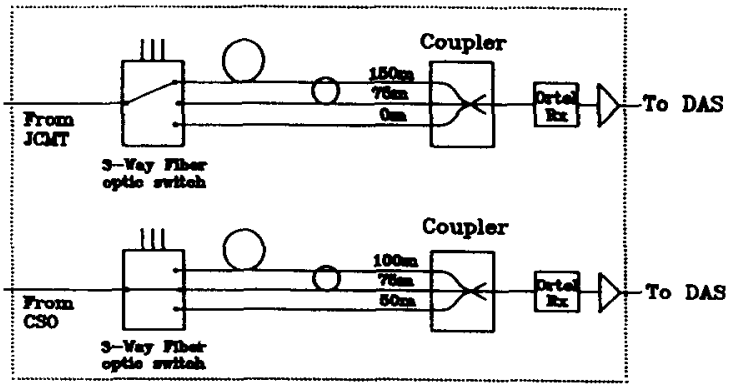

b)

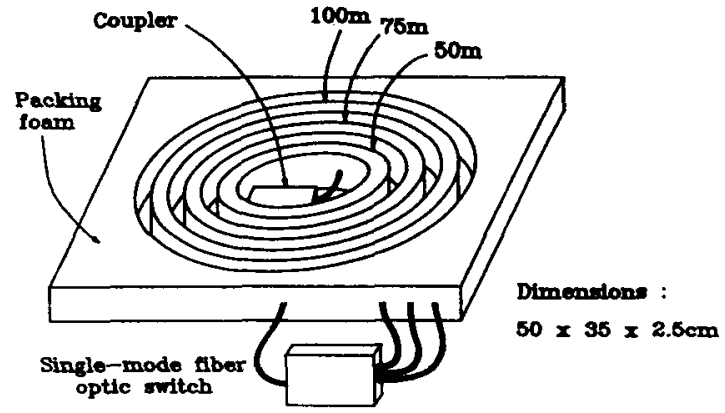

FIGURE I Delay line design

always below $1 \%$ if the white light fringe is kept in the central $15 \%$ of the lag window. The smallest lag window possible on the DAS is $800 \mathrm{~ns}$ wide and this then sets the delay step size at 120ns. These steps must cover the baseline range: of $\pm 550 \mathrm{~ns}$. Nine steps are needed to do this.

The nine steps were implemented as shown in Figure I(a). Two 10GHz bandwidth optical links (from Ortel) are used to transmit and receive the infrared light on which the IF is intensity modulated. Two three-way fiber optic, switches (BT\&D) and two 3-to-1 star couplers (Canstar) select the two lengths of fiber through which the light must pass. The fiber delays cover the range $\pm 500 \mathrm{~ns}$ in steps of $120 \mathrm{~ns}$. Fusion splices are used for the permanent joins in the fiber and FC-APC connectors are used elsewhere. These both have insertion losses of less than $0.1 \mathrm{~dB}$ and all back-reflection ratings are better than $-40 \mathrm{~dB}$.

Figure I(b) illustrates just how compact the use of fiber enabled the design to be. The packing foam was an ideal medium to contain and protect the fiber and optical components. Two such units, each with three lengths of delay, sit in the base of a 19 inch rack box with the two Ortel receivers and IF amplifiers The lines can be switched either manually or by a microcomputer that keeps track of the amount of delay required for a particular source at a particular time.

During the two runs to date of the JCMT-CSO interferometer, we have yet to experience any problems with the delay lines. Amplitude and phase stability are excellent. The linear dynamic range is very good and noise is more than $30 \mathrm{~dB}$ down on the nominal IF signal level. 